\title{
Molecular Symmetry Governs Surface Diffusion
}

\author{
Tobias Sonnleitner, Ingmar Swart, ${ }^{*}$ Niko Pavliček, Andreas Pöllmann, and Jascha Repp \\ Institute of Experimental and Applied Physics, University of Regensburg, 93053 Regensburg, Germany
}

(Received 8 June 2011; published 27 October 2011)

\begin{abstract}
In chemistry and physics symmetry principles are all important, for example, leading to the selection rules governing optical transitions. We have investigated the influence of the molecular symmetry on the surface potential landscape of molecules in the limit of weak molecule-substrate binding. For this purpose, the induced lateral motion of $\mathrm{Cu}$ (II)-tetraazaphthalocyanine molecules, for which four symmetry distinct isomers exist, on $\mathrm{NaCl}(100)$ was studied by scanning tunneling microscopy. This nonthermal diffusion induced by inelastic excitations is found to be qualitatively different for all four symmetry distinct isomers, demonstrating that symmetry governs the surface potential landscape.
\end{abstract}

PACS numbers: 68.35.Fx, 68.37.Ef, 73.61.Ng, 82.37.Gk

Symmetries govern many fields of chemistry and physics. In surface science chiral selectivity in surface structure formation is a prominent example of the relevance of symmetry principles $[1,2]$. Diffusion restricted to one dimension due to surface anisotropy can be explained by reduced substrate symmetry [3-7]. In a few experiments it has been found that a twofold symmetric molecule exhibits diffusion restricted to one dimension although the substrate was threefold rotationally symmetric [8-10]. This indicates the possible importance of the adsorbate symmetry for surface diffusion. Surprisingly, no systematic studies into this topic exist. In surface diffusion there are no such things as selection rules that forbid certain diffusion steps. This underlines the need for a deeper understanding of how the potential energy landscape, and hence surface diffusion, is influenced by molecular symmetry.

In this Letter we address the issue of how the surface potential landscape of molecules is influenced by the molecular symmetry. An ideal molecule to perform such studies is $\mathrm{Cu}(\mathrm{II})$-tetraazaphthalocyanine $(4 \mathrm{NCuPc})$, since for this molecule 4 symmetry distinct isomers $\left(C_{4 h}, D_{2 h}\right.$, $C_{2 v}$, and $C_{s}$ ) exist, which differ only in the position of $4 \mathrm{~N}$ atoms in the molecular frame. We mapped out the potential energy landscape by exciting individual molecules by inelastically tunneling electrons and analyzing the patterns of motion using scanning tunneling microscopy (STM). The lateral motion of all four isomers on an ultrathin insulating $\mathrm{NaCl}$ film was investigated. The interaction between $\pi$-conjugated molecules and insulators is expected to be rather weak [11]. In contrast to molecules on metals [5,12], in general very little is known about the diffusion of organic molecules on insulators [13]. We find that the nonthermal diffusion behavior induced by inelastic excitation of the four symmetry distinct isomers is qualitatively different, thereby unambiguously demonstrating the influence of adsorbate symmetry.

The experiments were carried out in a modified commercial low-temperature $(T=5 \mathrm{~K}) \quad$ ultrahigh vacuum $\left(p<10^{-10}\right.$ mbar) scanning tunneling microscope
(SPS-CREATEC). After cleaning a $\mathrm{Cu}(111)$ single crystal by several sputtering and annealing cycles, defect-free (100)-terminated $\mathrm{NaCl}$ bilayer islands were grown at room temperature [14]. After transferring the sample into the STM, 4NCuPc molecules were thermally evaporated at sample temperatures below $10 \mathrm{~K}$. Bias voltages refer to the sample voltage with respect to the tip. We used an electrochemically etched tungsten wire as the STM tip.

$4 \mathrm{NCuPc}$ is related to the well-known $\mathrm{Cu}(\mathrm{II})-$ phthalocyanine $(\mathrm{CuPc})$. On each of the four arms of $\mathrm{CuPc}$, one $\mathrm{C}-\mathrm{H}$ group in the outermost benzene ring is substituted by a $\mathrm{N}$ atom [inset of Fig. 1(a)]. There are two equivalent positions for the $\mathrm{N}$ atom on each arm, indicated by arrows. This gives rise to four symmetry distinct isomers $\left(C_{4 h}, D_{2 h}, C_{2 v}\right.$, and $\left.C_{s}\right)$ of $4 \mathrm{NCuPc}$. Upon adsorption, the symmetries of these four isomers are reduced to $C_{4}, C_{2}$, $C_{s}$, and $C_{1}$, respectively (see below) [15]. These symmetry labels will therefore be used throughout this report. Schematics showing how the $4 \mathrm{~N}$ atoms are distributed in each isomer are given in Fig. 1(c), top panel. It is important to realize that the $C_{4}, C_{2}$, and $C_{s}$ isomers all differ from the $C_{1}$ isomer only by interchanging the position of $1 \mathrm{~N}$ atom and a neighboring $\mathrm{C}-\mathrm{H}$ group in this large organic molecule. Gas-phase molecules with $C_{4 h}$ and $C_{s}$ symmetry are prochiral with respect to adsorption [16]; hence, there must exist two enantiomers of the $C_{4}$ and $C_{1}$ isomers on the surface (labeled $C_{4}^{\prime}$ and $C_{1}^{\prime}$ ) [2,17].

How does adsorption on a surface influence the molecular symmetry [18]? Irrespective of the substrate structure, in-surface-plane mirror symmetry as well as all rotational symmetries with axes that are not perpendicular to the surface plane are lost. Furthermore, the adsorbate-substrate system can have only out-of-surface-plane symmetry operations that both the molecule and the substrate possess. Of these symmetry operations only those remain for which the spatial position of the symmetry elements (rotation axis, mirror plane) of molecule and substrate coincide. Hence, it is of prime importance to determine the adsorption site and geometry. Provided that molecules adsorb in 

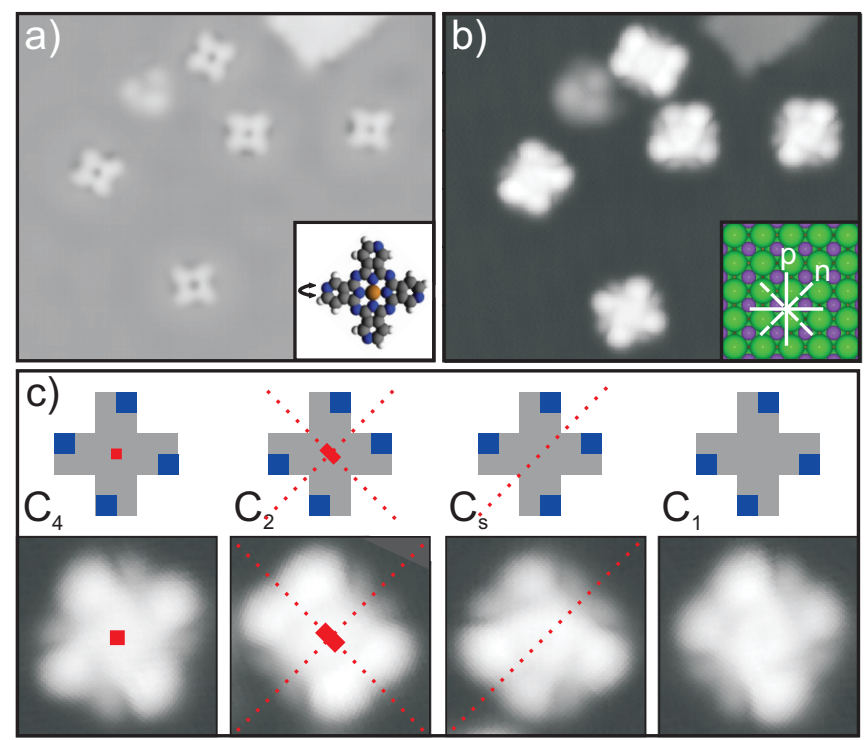

FIG. 1 (color). STM images and schematics of the four different 4NCuPc isomers. (a) Constant-current STM image acquired at $V=0.1 \mathrm{~V}$ and $I=5 \mathrm{pA}$, showing five molecules. The molecules appear crosslike, resembling their geometry $(140 \AA \times$ $120 \AA$ ). The inset shows a model of the $C_{4}$ isomer. Arrows indicate the two equivalent positions for the $\mathrm{N}$ atom. Gray, blue, white, and orange indicate $\mathrm{C}, \mathrm{N}, \mathrm{H}$, and $\mathrm{Cu}$ atoms, respectively. (b) Image acquired at a voltage corresponding to tunneling through the LUMO $(140 \AA \times 120 \AA, 5 \mathrm{pA}, 1.6 \mathrm{~V})$. The inset shows a model of the $\mathrm{NaCl}$ lattice, indicating the polar ( $p$, solid line) and nonpolar ( $n$, dashed lines) directions (green: $\mathrm{Cl}$, purple: $\mathrm{Na}$; not drawn to scale). (c) Orbital image (bottom) and schematic (top) of the $C_{4}, C_{2}, C_{s}$, and $C_{1}$ isomers (left to right), respectively. In the orbital images, the molecules are oriented in the same way as in the schematic. Blue squares indicate the position of the outer $\mathrm{N}$ atoms in the carbon framework. A red square (rectangle) indicates a fourfold (twofold) rotation axis. Red dotted lines indicate mirror planes perpendicular to the molecular plane. For $C_{2}$ isomers the mirror planes do not coincide with mirror planes of the $\mathrm{NaCl}$ film and are therefore lost (see Fig. 2).

high-symmetry sites, the atomic structure of the $\mathrm{NaCl}(100)$ film does not reduce the rotational symmetry of $4 \mathrm{NCuPc}$ isomers, since it is compatible with both a twofold and a fourfold rotation. This holds true irrespective of the azimuthal angle of adsorption (i.e., the in-surface-plane rotation). If the overall symmetry of the adsorbate-substrate system is lower than that of the clean surface, then this has direct consequences for the degeneracy of adsorption geometries. If mirror planes perpendicular to the surface exist for both the substrate and the adsorbate, but they do not coincide [see $C_{2}$ molecule in Fig. 1(b)], an application of this symmetry operation of the substrate (mirroring) will lead to an adsorption geometry that is equivalent in the sense that it will be associated with the same adsorption energy, but different with respect to the observed azimuthal angle. Thus, this situation must be accompanied with a degeneracy of adsorption geometries. The same holds true for any rotational symmetry operation of the substrate that is lost upon adsorption. Such degeneracies of adsorption geometries are directly reflected in the potential energy landscape [15].

To study the interplay between symmetries and diffusion, we mapped out the potential energy landscape as follows. The energy required to overcome the diffusion barrier was supplied to individual molecules by inelastically tunneling electrons $[19,20]$. The excitation procedure is illustrated in Fig. 2(a). The tip is positioned above the center of a molecule (position indicated by the magenta colored dots) to not favor any direction [20]. The feedback loop is interrupted and a bias voltage below $-2 \mathrm{~V}$ is applied to the sample. The bias voltage is kept constant until a sudden drop in the current occurs, signaling a lateral motion [21].

This experiment was performed tens of times for each of the four symmetry distinct isomers, allowing the construction of isomer specific movies (see Supplemental Material [22]). From each of these movies, a pattern of adsorption positions was determined with respect to a marker molecule. The number of equivalent adsorption sites per surface unit cell can be directly obtained from these patterns. The orientation of the molecule after each hop was determined using the fact that the edges of $\mathrm{NaCl}$ islands grown on $\mathrm{Cu}$ (111) are strictly nonpolar [23]. As discussed above, detailed knowledge regarding the adsorption site is required, which we have determined for all four isomers by using coevaporated $\mathrm{Au}$ atoms as markers (Au atoms adsorb on top of $\mathrm{Cl}$ atoms [24]). The spatial patterns and histograms of the observed azimuthal angles of all four isomers are given in Fig. 2.

In STM images acquired at low bias voltages [Fig. 1(a)], all $4 \mathrm{NCuPc}$ molecules appear similar and have a crosslike shape, resembling the geometrical structure of the molecule. When increasing the bias voltage to values corresponding to tunneling resonantly into the lowest unoccupied molecular orbital (LUMO), four symmetry distinct isomers are observed [Fig. 1(b)]. These have the symmetries expected from the basic considerations outlined above, allowing direct isomer identification. It is found that for all four isomers, there is one bigger and one smaller lobe localized on each of the four arms. A comparison to density functional theory calculations of free molecules shows that the smaller lobes are located at the position of the $\mathrm{N}$ atoms. Below, we will sequentially discuss the current-induced diffusion of the four isomers in order of decreasing symmetry.

As can be seen in movie M1 [22], the $C_{4}$ isomer moves in two dimensions. The pattern of observed positions [cf. movie M2 in [22] and Fig. 2(c)] can be easily brought in congruence with the $\mathrm{NaCl}$ surface lattice and exhibits two adsorption sites per surface unit cell. From the experiments using $\mathrm{Au}$ atoms as markers, these sites are identified as on top of $\mathrm{Cl}$ and $\mathrm{Na}$ [dark and light blue diamonds in Fig. 2(c), 

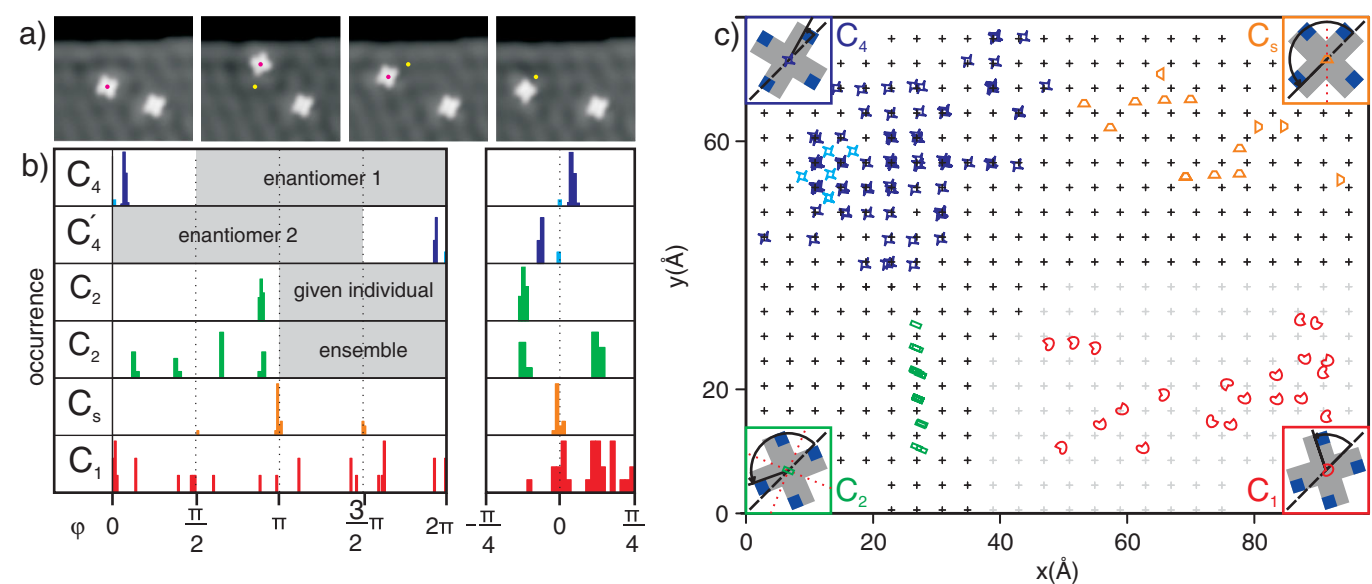

FIG. 2 (color). (a) Exemplary images out of a series in which the current-induced motion of the $C_{1}$ isomer was studied. Magenta and yellow dots indicate the positions of current injection and the previous position of the molecule, respectively. Imaging parameters: $V=0.1 \mathrm{~V}, I=5 \mathrm{pA}$. (b) Histograms of occurrences of azimuthal angles between a molecular arm and the nonpolar direction of the $\mathrm{NaCl}(100)$ lattice. The angles are defined as indicated by the insets shown in (c). In the right panel the same data are reduced to one quadrant taking into account the fourfold symmetry of the $\mathrm{NaCl}$ lattice. (c) Patterns of motion for the different isomers. Blue, green, orange, and red symbols indicate the relative positions of individual $C_{4}, C_{2}, C_{s}$, and $C_{1}$ isomers with respect to a marker molecule, respectively. Black crosses indicate $\mathrm{Cl}$ sites in the $\mathrm{NaCl}(100)$-surface lattice. Light gray crosses indicate equivalent lattice sites. For the $\mathrm{C}_{4}$ isomer, data acquired on two molecules are combined. The black dashed lines in the insets shown in (c) indicate one of the nonpolar directions of the $\mathrm{NaCl}$ lattice. The red dotted lines indicate the mirror planes of the isomers perpendicular to the molecular plane. For $C_{2}$ isomers the mirror planes do not coincide with mirror planes of the $\mathrm{NaCl}$ film and are therefore lost.

respectively]. From the ratio of the observed sites, we can conclude that the on-top $\mathrm{Cl}$ site is lower in energy. Because of the fourfold rotational symmetry of the $C_{4}$ isomer, the azimuthal angle is only defined in the interval 0 to $\pi / 2$. For each of the two adsorption sites only one angle is observed. However, a change in the adsorption site is accompanied by a rotation over $13^{\circ}$. Interestingly, in the movie the molecule laterally moved several times between different metastable positions before it moved to the most stable position and the corresponding orientation. This can only be explained if the barrier for rotation is higher than the barrier for lateral movement. Since gas-phase molecules with $C_{4 h}$ symmetry are prochiral with respect to adsorption two enantiomers, $C_{4}$ and $C_{4}^{\prime}$, of this isomer should be observed on the surface. The azimuthal angle of $C_{4}^{\prime}$ must be the reverse of the one of $C_{4}$ with respect to one of the mirror planes of $\mathrm{NaCl}(100)$, since the application of such a symmetry operation transforms one enantiomer into the other. We observe both enantiomers on the surface with the expected relation between the azimuthal angles [Fig. 2(b)]. This establishes that the molecular symmetry plays a decisive role in the potential energy landscape.

The movie of the $C_{2}$ isomer (movie M3 [22]) shows that this isomer moves along a straight line. The analysis demonstrates that the $C_{2}$ isomer performs a one-dimensional random walk on a grid [movie M4 [22] and green rectangles in Fig. 2(c)]. There is one adsorption site per $\mathrm{NaCl}(100)$ surface unit cell. The site determination shows that the molecule is adsorbed in a bridge site. During the motion, the azimuthal angle always remains the same; i.e., the molecule does not rotate. Interestingly, this angle is not aligned with one of the mirror planes of the $\mathrm{NaCl}(100)$ surface [see Fig. 1(b) and inset in Fig. 2(c)]. Hence, the resulting symmetry of the adsorbate-substrate system is $C_{2}$. As discussed above, this implies that there must exist two degenerate adsorption geometries with respect to the azimuthal angle. In addition, there must exist another pair of angles differing by $\pi / 2$ for the nonequivalent bridge site. This gives a total of four adsorption geometries with inequivalent angles but with the same energy. Indeed, by analyzing the adsorption geometries of an ensemble of $C_{2}$ isomers, all four angles are observed [cf. Fig. 2(b)]. Since we find only one angle for a given individual molecule, the energy barrier for rotation must be significantly larger than for lateral motion. As the rotational orientation also determines the potential energy landscape for lateral movement, combined with the fact that the molecule is adsorbed in a twofold rotationally symmetric bridge site, leads to diffusion confined to one dimension [8,9]. Hence, the 1D diffusion of the $C_{2}$ isomer is a direct consequence of the adsorbate-substrate symmetry.

The movie of the $C_{s}$ isomer (movie M5 [22]) demonstrates that this isomer hops on a 2D square lattice [movie M6 [22] and orange triangles in Fig. 2(c)] with only one adsorption site per surface unit cell. The resonance images in the movie show that this isomer can rotate. Only four distinct orientations differing by $\pi / 2$ are observed, with the arms of the molecule always aligned with the nonpolar direction of the $\mathrm{NaCl}(100)$ surface [Fig. 2(b)]. Hence, the mirror plane of the molecule aligns with one of the mirror 
planes of the surface. From the above it can be concluded that the molecule must be adsorbed on top of either a $\mathrm{Na}$ or a $\mathrm{Cl}$ atom. Together with the site determination, we find that the center of this isomer is located directly on top of a $\mathrm{Na}$ atom.

As can be seen in movie M7 [22], the $C_{1}$ isomer hops in two dimensions and many adsorption orientations are observed. In contrast to all other isomers, no preferential adsorption site is apparent from the analysis of the pattern of motion [movie M8 [22] and Fig. 2(c)]. Hence, no absolute site determination can be done. This implies that the potential energy surface exhibits multiple local minima per surface unit cell for this isomer. This is consistent with the observation of many different azimuthal orientations [movie M7 [22] and Fig. 2(b)].

Since in our study the excitation comes about by tunneling into or out of one of the molecular resonances, in principle, the symmetry of that stimulus could restrict the molecular motion to certain symmetry. However, we believe this to be extremely unlikely. We expect that the vibrational excitation has to be distributed inside the molecule to other modes before it can result in molecular motion. We therefore do not expect that the excitation mechanism can explain the qualitative differences in observed patterns. Instead, we believe that the latter directly reflects the difference in the potential energy landscape. From a comparison of all the different observed patterns it becomes apparent that the observed binding sites and angles show a direct relationship to the symmetry: this implies that beside the structural properties also the adsorbate symmetry is decisive for the surface potential landscape. The symmetry-related behavior of the adsorption and lateral motion cannot be generated solely by longrange van der Waals (vdW) forces that lack directionality. Our results can be rationalized by assuming that there is a polar interaction between the nitrogen atoms in the molecule and the substrate. In addition, vdW forces contribute significantly to the overall adsorption energy. An accurate theoretical description of the present system will therefore require a correct treatment of these forces, making the results described in this Letter a benchmark system for the theoretical modeling of systems in which $\mathrm{vdW}$ forces play a dominant role [25].

In summary, we have investigated the influence of symmetry on the surface potential landscape of $\pi$-conjugated molecules on an insulating surface. We find that the behavior of the four symmetry distinct isomers of $4 \mathrm{NCuPc}$ is qualitatively different. For one isomer $\left(C_{1}\right)$ there is no apparent preferred adsorption site, whereas for others one $\left(C_{s}\right.$ and $\left.C_{2}\right)$ or two $\left(C_{4}\right)$ preferred sites can be observed. Some isomers can rotate $\left(C_{1}\right.$ and $\left.C_{s}\right)$, whereas another isomer does not $\left(C_{2}\right)$. It should be noted that the $C_{4}, C_{2}$, and $C_{s}$ isomers all differ from the $C_{1}$ isomer only by interchanging the position of one $\mathrm{N}$ atom and a neighboring $\mathrm{C}-\mathrm{H}$ group in a large organic molecule.
Although in diffusion no generally applicable simple selection rules can be formulated, as the surface potential landscape is always given by the interplay of adsorbate and substrate, our study establishes that the adsorbate symmetry plays a decisive role.

We thank K. S. Thygesen and T. Markussen for discussions. Financial support from the Volkswagen Foundation (Lichtenberg program), the Deutsche Forschungsgemeinschaft (GRK 1570) and the Nederlandse Organisatie voor Wetenschappelijk Onderzoek (Rubicon-grant 680.50.0907) is acknowledged.

*Ingmar.Swart@physik.uni-regensburg.de

[1] R. Fasel et al., Nature (London) 439, 449 (2006).

[2] K.H. Ernst, in Supramolecular Chirality, Topics in Current Chemistry Vol. 265 (Springer-Verlag, Berlin, Heidelberg, New York, 2006) pp. 209-252.

[3] E. Ganz et al., Phys. Rev. Lett. 68, 1567 (1992).

[4] J. Weckesser et al., J. Chem. Phys. 110, 5351 (1999).

[5] J. V. Barth, Surf. Sci. Rep. 40, 75 (2000).

[6] M. Schunack et al., Phys. Rev. Lett. 88, 156102 (2002).

[7] R. Otero et al., Nature Mater. 3, 779 (2004).

[8] K. Y. Kwon et al., Phys. Rev. Lett. 95, 166101 (2005).

[9] K. L. Wong et al., Science 315, 1391 (2007).

[10] M. Eichberger et al., Nano Lett. 8, 4608 (2008).

[11] J. Repp et al., Phys. Rev. Lett. 94, 026803 (2005).

[12] F. Rosei et al., Prog. Surf. Sci. 71, 95 (2003).

[13] B. Such et al., ACS Nano 4, 3429 (2010).

[14] R. Bennewitz et al., Surf. Sci. 438, 289 (1999).

[15] The underlying copper substrate is not taken into account, since it should not strongly affect the symmetry of molecules adsorbed on a bilayer of $\mathrm{NaCl}$. The results presented throughout this Letter support this assumption.

[16] A. G. Mark, M. Forster, and R. Raval, Tetrahedron: Asymmetry 21, 1125 (2010).

[17] M. Parschau et al., Angew. Chem., Int. Ed. 48, 4065 (2009).

[18] R. Raval, Chem. Soc. Rev. 38, 707 (2009).

[19] T. Komeda et al., Science 295, 2055 (2002).

[20] M. Ohara, Y. Kim, and M. Kawai, Phys. Rev. B 78, 201405 (2008).

[21] Comparing images and STS data before and after the excitation shows that the molecules are not damaged during this procedure. In addition, no defects in the $\mathrm{NaCl}$ lattice are observed after the manipulation.

[22] See Supplemental Material at http://link.aps.org/ supplemental/10.1103/PhysRevLett.107.186103 for the isomer specific movies. For each isomer, there is one movie showing resonance and low voltage images and a second one with the curvature of the latter, superimposed with the motion pattern.

[23] J. Repp and G. Meyer, Appl. Phys. A 85, 399 (2006).

[24] J. Repp, G. Meyer, F. E. Olsson, and M. Persson, Science 305, 493 (2004).

[25] The molecules are negatively charged. 